BNWL-1931

Special Distribution in Category UC-70

\title{
Diets of Black-Tailed Hares on the Hanford Reservation
}

April 1975

Prepared for the U.S. Energy

Research and Development Administration under Contract E(45-1):1830 


\section{NOTICE}

This report was prepared as an account of work sponsored by the United States Government. Neither the United States nor the United States Energy Research and Development Administration, nor any of their employees. nor any of their contractors, subcontractors, or their employees, makes any warranty, express or implied, or assumes any legal liability of responsibility for the accuracy, completeness or usefulness of any information. apparatus, product or process disclosed, or represents that its use would not infringe privately owned rights.

\section{PACIFIC NORTHWEST LABORATORY}

operated by

BATTELLE

for the

U.S. ENERGY RESEARCH AND DEVELOPMENT ADMINISTRATION

Under Contract E(45-1)-1830

Printed in the United States of America

Available from

National Technical Information Service

U.S. Department of Commerce

5285 Port Royal Road

Springfield, Virginia 22751

Price: Printed Copy \$5.00; Microliche \$2.25 


\section{Diets of Black-Tailed Hares on the Hanford Reservation}

April 1975

D. W. Uresk, J. F. Cline and W. H. Rickard Ecosystems Department

This report summarizes work sponsored by The Atlantic Richfield Hanford Company under United States Energy Research and Development Administration

Contract AT(45-1)-2130

BATTELLE

Pacific Northwest Laboratories

Richland, Washington 99352 


\section{TABLE OF CONTENTS}

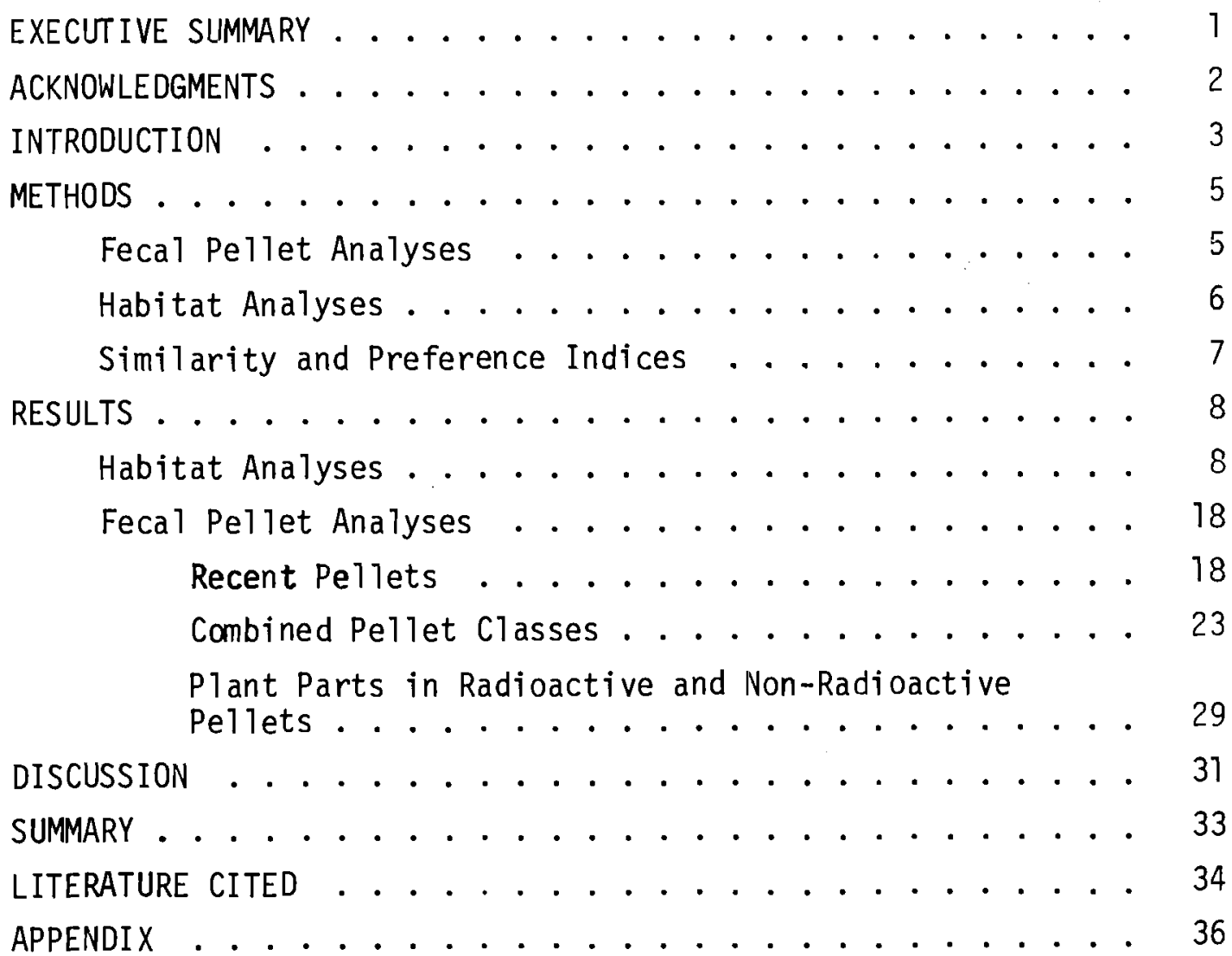




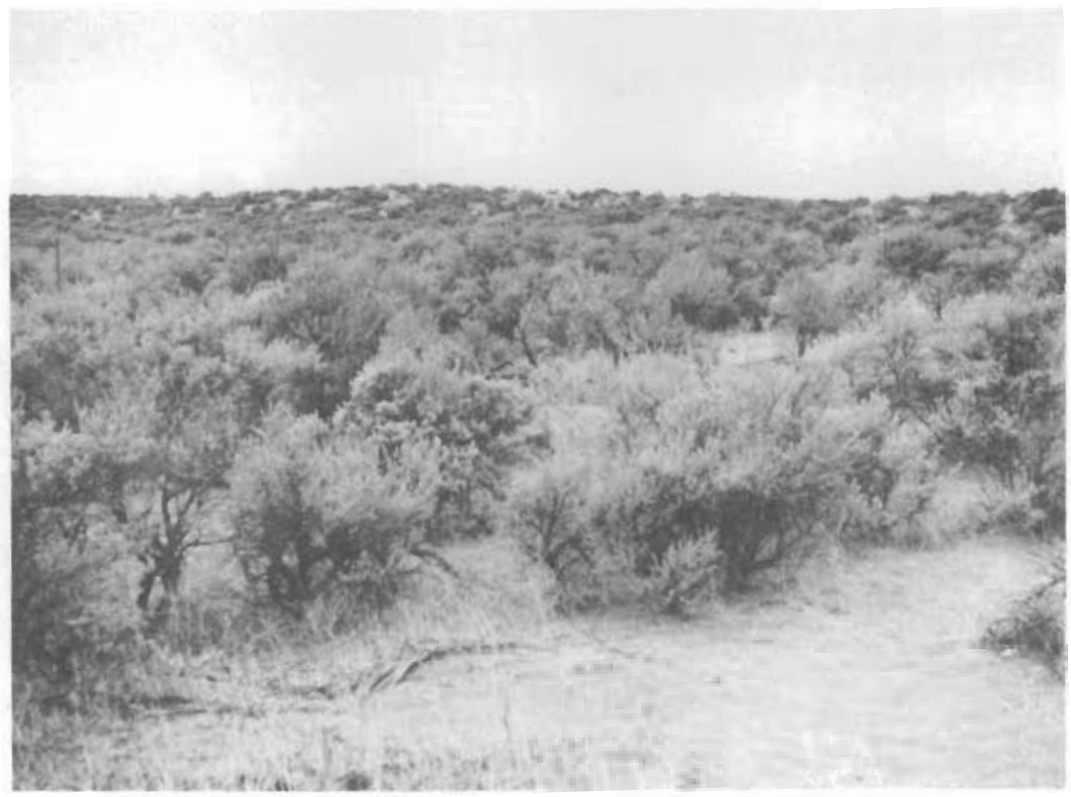

FIGURE 1. Big Sagebrush (Artemisia tridentata) Plant Community Common to the B-C Cribs Area

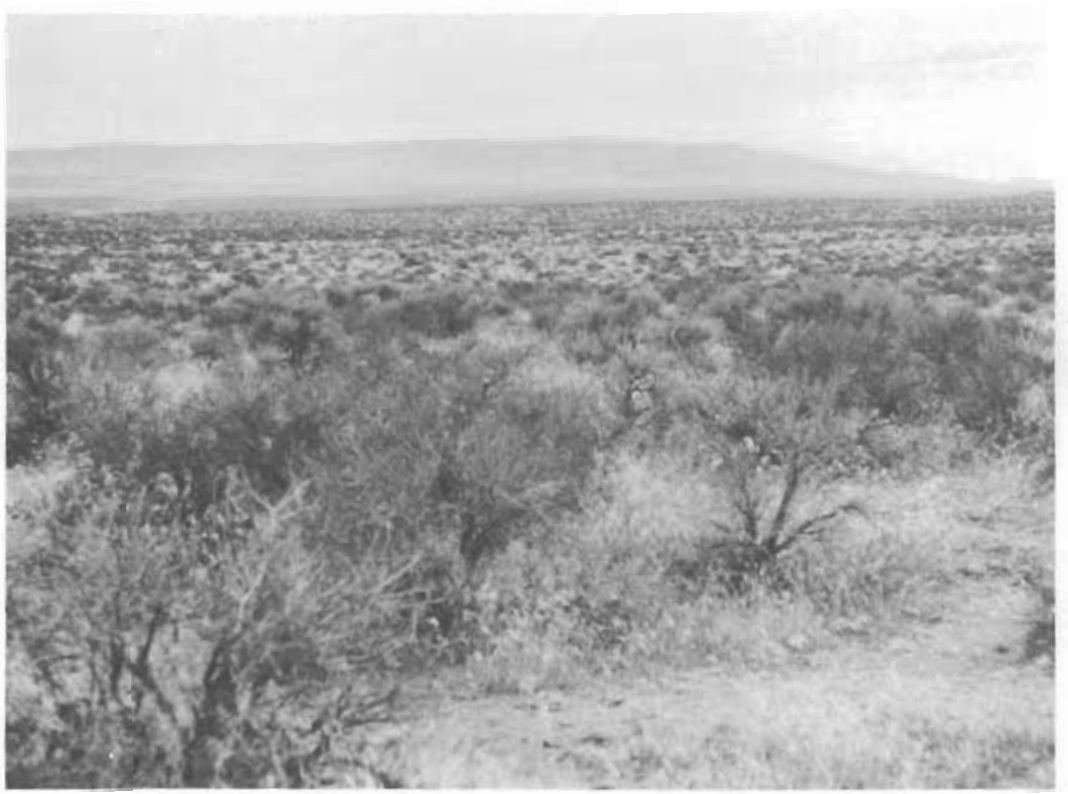

FIGURE 2. Bitterbrush (Pursha tridentata) Plant Community Common to the Wye-Barricade Area 
BNWL -1931

\section{EXECUTIVE SUMMARY}

A fecal pellet analyses showed that black-tailed hares (jackrabbits) were selective in plants chosen as food. The most abundant herbaceous plant, cheatgrass, was not found in the pellets. Sagebrush and bitterbrush, woody plants, were not an important part of the hares' diet. Forbs, rabbitbrush and certain grass species were preferred foods. 


\section{ACKNOWLEDGMENTS}

The authors thank $\mathrm{Dr}$. L. E. Rogers for his help with the diet analyses and helpful suggestions. Thanks are also extended to Dr. R. 0. Gilber:, M. A. Wise, and L. E. Rendall for technical help and data analyses. W: extend our thanks to B. J. Rathjen for typing this manuscript.

We wish to thank the Envirormental Engineering Section of Atlantic Richfield Hanford Company for sponsoring this study. 
BNWL-1931

\section{DIETS OF BLACK-TAILED HARES ON THE HANFORD RESERVATION}

\section{INTRODUCTION}

The purpose of this investigation was to examine the fecal pellets dropped by black-tailed hares (Lepus californicus), as a means to determine the kinds of plants eaten.

Two widely separated and different plant communities were selected as study areas. One dominated by big sagebrush (Artemisia tridentata) was located adjacent to the B-C Cribs Controlled area and the other dominated by bitterbrush (Pursha tridentata) was located in the vicinity of the WyeBarricade (Figure 1).

Al though cheatgrass, Bromus tectorum, is the most abundant herbaceous plant observed in the field, it did not appear in the fecal pellets examined. The plant species most often associated with fecal pellets were yarrow, Achillea millifolium; turpentine cymopterus, Cymopterus terebinthinus; and Erigeron sp. All are relatively scarce in the hare's habitat. The shrub species most often represented in fecal pellets was rabbitbrush, Chrysothamnus nauseosus, which was common in habitat. 


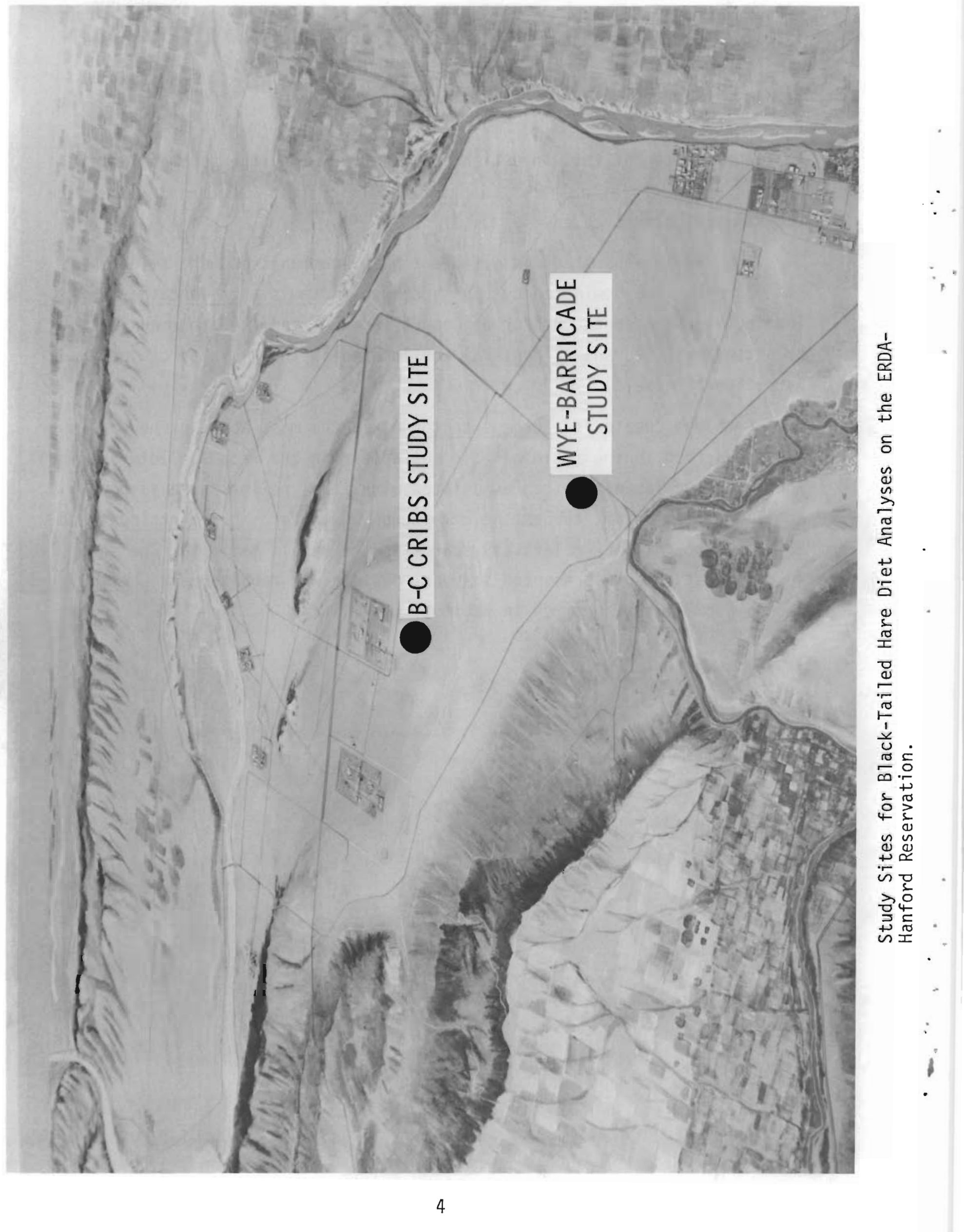


BNWL-1931

\section{METHODS}

The experimental design for collecting fecal pellets of hares consisted of two replicated sites located at random in each of two habitat types within the B-C Cribs Control zone and near the Wye-Barricade. Within each replicate two parallel transects $(1 \mathrm{~m} \times 50 \mathrm{~m})$ were spaced 60 meters apart. Each transect was subdivided into two lengths -1 to 25 meters and 25 to 50 meters. A11 pellets were collected from the two subdivisions of each transect and designated as two samples. This procedure was repeated for all transects, making a total of four samples for each replicate. The number of pellets in the samples by classes ranged from 20 to 1026. A total of 6712 pellets was analyzed in the B-C Cribs Controlled zone and 2871 in the Wye-Barricade site.

The experimental design for plant habitat comparisons was a nested factorial design and all tests, including analysis of variance, follow Snedecor and Cochran (1967). For multiple comparisons, a Bonferroni ttest was used as outlined by Miller (1966).

\section{FECAL PELLET ANALYSES}

Fecal pellets were plucked from the ground by hand and categorized into four general age classes: (1) recent pellets, less than 1 year old and green in color; (2) brown pellets, more than 1 year old; (3) gray-brown pellets, eroded in size and shape, several years old; and (4) a general category consisting of mixtures of classes 2 and 3 . Additional fecal pellets were collected and categorized as radioactive. Pellets were dried at $70^{\circ} \mathrm{C}$ for 48 hours and ground in a Wiley mill $(1 \mathrm{~mm}$ screen) to make a homogeneous sample. All sampling was conducted during April 1974.

Five slides were prepared for each of the four samples by classes after the contents had been washed over a $0.1 \mathrm{~mm}$ screen (Sparks and Malechek, 1968). Slides were prepared with Hertwig's solution and Hoyer's solution (Baumgartner and Martin, 1939; Hansen and Flinders, 1969) and dried for approximately 72 hours at $60^{\circ} \mathrm{C}$. 
Identification of plant fragments was made by microscopic examination of fecal material on the basis of structural modifications of epidermal cells when compared to reference plant material prepared from specimens identified and collected from the study areas. Twenty microscope fields were examined on each slide and all recognizable fragments recorded. Frequency of occurrence was determined by dividing the number of microscope views in which a given species occurred by the total number of views $\times 100$ (Curtis and McIntosh, 1950). This value of frequency of a given species was divided by the total of the frequency values for all species and expressed as percent frequency.

\section{HABITAT ANALYSES}

Vegetational analyses were made of species composition, canopycover provided by each species, and frequency of occurrence. Two replicated sites were examined adjacent to the B-C Cribs Controlled zone, and also in the Wye-Barricade area. The area adjacent to the B-C Cribs Controlled zone was utilized only for vegetational analyses. Each replicate adjacent to the B-C Cribs Controlled zone consisted of four parallel line transects each $50 \mathrm{~m}$ long and $30 \mathrm{~m}$ apart while vegetational measurements were on the same two pellet transects for each transect in the WyeBarricade habitat. The canopy-cover method of vegetational analysis developed by Daubenmire (1959) was used for herbaceous taxa. Canopycover was estimated using the following cover-class categories: class 1 $(1-5 \%)$, class $2(6-15 \%)$, class $3(16-25 \%)$, class $4(26-50 \%)$, class 5 (51-75\%), and class $6(76-100 \%)$, by ocularly examining fifty $(2 \times 5 \mathrm{dm}$, $0.1 \mathrm{~m}^{2}$ ) plots systematically spaced at 1 meter intervals along each $50 \mathrm{~m}$ line transect. For summarization of the canopy-cover for each line transect, an average was obtained by summing the median percent value for each size class for each $2 \times 5 \mathrm{dm}$ plot and dividing by 50 . Frequency of occurrence of a given species was divided by the total of the frequency values for all species and expressed as percent frequency for habitat analyses. All field sampling of the vegetation was conducted during Apri 11974.

The density of shrubs was determined by counting all shrubs rooted within a $10 \times 50 \mathrm{~m}$ plot centrally located in each replicated study site. 
Each shrub was measured for long and short diameter and for height above ground.

\section{SIMILARITY AND PREFERENCE INDICES}

Similarity indices were determined by using Kulczynski's mathematic expression for similarity (0osting, 1956):

$$
S I=\frac{2 w}{a+b}(100)
$$

where $w=$ the lowest amount of an item shared by two categories, and $a+b=$ the total amount of an item shared by these two categories. Similarity indices were calculated for pellet and for habitat analyses to determine the degree of association.

Preference indices were calculated by dividing the percent frequency in the diet by percent frequency in the habitat (Krueger, 1972). Data collected in the field as frequency of occurrence (Hyder et al., 1965) was converted to percent frequency by species at a base of 100 or as a percentage of the total frequency of all species. The objective of this procedure is to put both the plants in the feces and plants in the field in the same relative proportions. Frequency of occurrence may be different for two areas, but the relative proportions from which the animal is selecting may be the same. Putting it on this basis, relative preference indices may be determined. As used here, the preference indices are regarded only as a guide to food preferences rather than absolute values. 


\section{RESULTS}

\section{HABITAT ANALYSES}

Shrubs are important to black-tailed hares. They provide protection from predators and shelter from adverse weather conditions. Shrub cover (length $x$ width $x$ density) averaged 34 and $44 \%$ adjacent to the $B-C$ Cribs Controlled area and Wye-Barricade habitats, respectively (Table 1).

TABLE 1. Canopy cover (\%) provided by shrubs at B-C Cribs and Wye-Barricade habitats.

\begin{tabular}{|c|c|c|c|c|c|c|}
\hline \multirow[b]{2}{*}{ Plant Species } & \multicolumn{3}{|c|}{ B-C Cribs } & \multicolumn{3}{|c|}{ Wye-Barricade } \\
\hline & $\overline{\operatorname{Rep} 1}$ & $\operatorname{Rep} 2$ & Avg. & Rep 1 & $\operatorname{Rep} 2$ & Avg. \\
\hline Artemisia & 32 & 35 & 34 & 6 & 12 & 9 \\
\hline Chrysothamnus & 0 & 1 & 1 & 6 & 3 & 5 \\
\hline Purshia & $\underline{0}$ & $\underline{0}$ & $\underline{0}$ & $\underline{34}$ & $\underline{25}$ & $\underline{30}$ \\
\hline Total & 32 & 36 & 35 & 46 & 40 & 44 \\
\hline
\end{tabular}

Thus, a significant difference $(P \leq 0.01)$ in shrub cover occurred between these two habitats (Table 2). This is primarily due to the occurrence of bitterbrush at the Wye-Barricade site. However, the canopy area provided by individual sagebrush plants was lower $(P \leq 0.01)$ at the $B-C$ Cribs site compared to sagebrush within the Wye-Barricade habitat. The average number of shrubs in the B-C Cribs habitat was $188 / 500 \mathrm{~m}^{2}$ as compared to $90 / 500 \mathrm{~m}^{2}$ in the Wye-Barricade habitat (Table 3). The more

TABLE 3. Density of shrubs (numbers per $500 \mathrm{~m}^{2}$ ) at the B-C Cribs and Wye-Barricade habitats.

\begin{tabular}{|c|c|c|c|c|c|c|}
\hline \multirow[b]{2}{*}{ Plant Species } & \multicolumn{3}{|c|}{ B-C Cribs } & \multicolumn{3}{|c|}{ Wye-Barricade } \\
\hline & Rep 1 & $\operatorname{Rep} 2$ & $\overline{A v g .}$ & $\operatorname{Rep~} 1$ & $\operatorname{Rep} 2$ & Avg. \\
\hline Artemisia & 113 & 259 & 186 & 20 & 23 & 22 \\
\hline Chrys othamnus & 0 & 4 & 2 & 22 & 11 & 17 \\
\hline Purshia & 0 & 0 & 0 & 67 & 35 & 51 \\
\hline Total & 113 & 263 & 188 & 109 & 69 & 90 \\
\hline
\end{tabular}


TABLE 2. Summary of canopy area (1ength $\times$ width, $\mathrm{cm}^{2}$ ) for shrubs located at B-C Cribs and Wye-Barricade habitats.

\begin{tabular}{|c|c|c|c|c|c|c|c|c|c|c|}
\hline \multirow[b]{2}{*}{ Plant Species } & \multicolumn{4}{|c|}{ Rep 1} & \multicolumn{3}{|c|}{ Rep 2} & \multicolumn{3}{|c|}{$\operatorname{Rep} 1+\operatorname{Rep} 2$} \\
\hline & $n$ & $\bar{X} \pm$ & & C.V.1/ & $n$ & $\mathrm{X} \pm \mathrm{SE}$ & C.V. & $n$ & $\bar{X} \pm S E$ & C.V. \\
\hline \multicolumn{11}{|c|}{ B-C Cribs } \\
\hline
\end{tabular}

\section{Statistical Treatment}

\section{Treatment}

B-C Cribs (a11 shrubs) vs. Wye-barricade (a11 shrubs) Replicates within habitats.

B-C Cribs (ㅁ․ tridentata) vs. Wye-barricade (․․ tridentata) Replicates within habitats.

\section{Significance}

$P<.01 \quad S$

$P \leq .001 \quad S$

$P \leq .01 \quad S$

$P \leq .001 \quad S$

1/Sample size, mean \pm standard error of the mean and coefficient of variation. 
dense stand of shrubs did not increase ground cover at the B-C Cribs habitat. Bitterbrush has a different growth form than sagebrush, providing more cover per shrub. Heights of sagebrush and bitterbrush were similar at both sites, averaging 85-88 cm (Table 4). Rabbitbrush was

TABLE 4. Average height $(\mathrm{cm})$ and length $x$ width $\left(\mathrm{cm}^{2}\right)$ of shrubs at the B-C Cribs and Wye-Barricade habitats.

\begin{tabular}{|c|c|c|c|c|}
\hline \multirow[b]{2}{*}{ Plant Species } & \multicolumn{2}{|c|}{ B-C Cribs } & \multicolumn{2}{|c|}{ Wye-Barricade } \\
\hline & $\overline{\mathrm{Ht}}$. & $L \times W$ & $\mathrm{Ht}$. & $L \times W$ \\
\hline Artemisia & 85 & 8991 & 87 & 21000 \\
\hline Chrysothamnus & 51 & 7285 & 58 & 14007 \\
\hline Purshia & -- & -- & 88 & 28930 \\
\hline All shrubs & 85 & 8973 & 83 & 23483 \\
\hline
\end{tabular}

much shorter in stature, ranging between 51 and $58 \mathrm{~cm}$. There were statistically significant differences $(P \geq 0.05)$ in shrub heights between sites (Table 5).

The total cover provided by herbaceous species was approximately $20 \%$ higher in the Wye-Barricade habitat than that associated adjacent to the B-C Cribs Controlled area (Table 6). The difference was significant at the $P \leq 0.01$ leve1 (Table 7).

When excluding the cheatgrass from the total cover, both sites had approximately the same $(P>0.05)$ amount of cover (Table 8$)$. Cheatgrass made up 35 and $15 \%(P \leq 0.01)$ of the total cover on the Wye-Barricade and $B-C$ Cribs habitat, respectively (Table 9 ).

The coefficient of variation is commonly used as a measure of variability. Excessive variability is sometimes present even though the standard errors of the mean may be small. The coefficient of variation (C.V.) is a useful measurement for comparing variability when the measurements are in different units. Since coefficients of variation are expressed as proportions or percentages and are divorced from units, they can be compared directly. 
BNWL- 1931

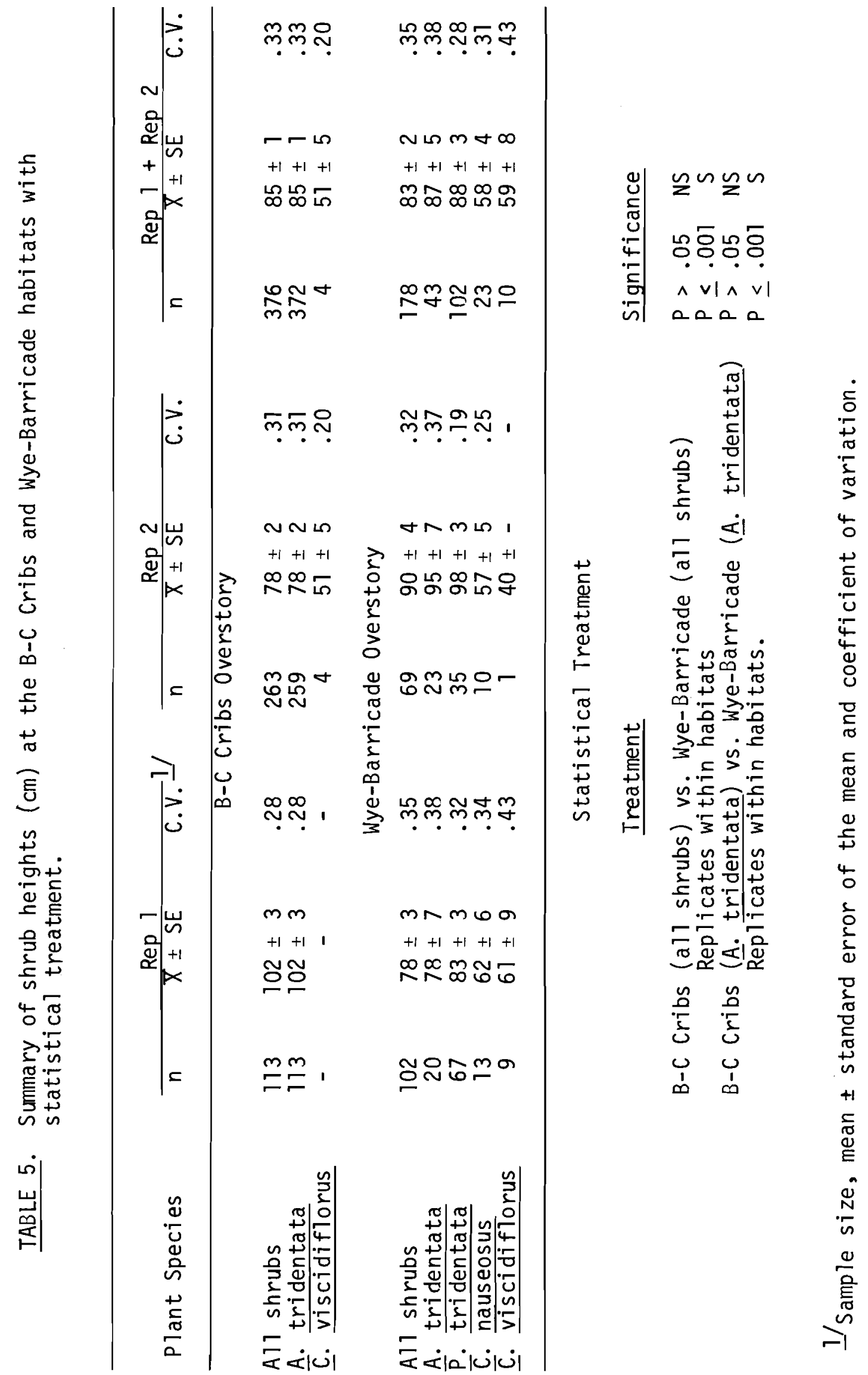


TABLE 6. Cover of herbaceous plants by species at the B-C Cribs and WyeBarricade habitats expressed as percentage of ground cover.

\begin{tabular}{|c|c|c|c|c|c|c|}
\hline \multirow[b]{2}{*}{ Plant Species } & \multicolumn{3}{|c|}{$B-C$ Cribs } & \multicolumn{3}{|c|}{ Wye-Barricade } \\
\hline & $\overline{\operatorname{Rep}}$ & $\overline{\operatorname{Rep}}$ & Avg. & $\overline{\operatorname{Rep}}$ & $\operatorname{Rep}$ & Avg. \\
\hline & \multicolumn{3}{|c|}{$\ldots-$ Percent $-\cdots$} & \multicolumn{3}{|c|}{$\ldots-$ Percent $\ldots-\cdots$} \\
\hline \multicolumn{7}{|l|}{ Grasses } \\
\hline B. tectorum & 45 & 34 & 40 & 61 & 69 & 65 \\
\hline $\bar{F}$. octoflora & 6 & 17 & 11 & $<1$ & 0 & $<1$ \\
\hline P. Sandbergii & 7 & 2 & 5 & 11 & 8 & 10 \\
\hline $\bar{S} . \overline{\text { comata }}$ & - & - & - & 0 & $<1$ & $<1$ \\
\hline Total & 58 & 53 & 56 & 72 & 77 & 75 \\
\hline \multicolumn{7}{|l|}{ Forbs } \\
\hline D. pinnata & 25 & 19 & 22 & $<1$ & $<1$ & $<1$ \\
\hline M. gracilis & 8 & 10 & 9 & 6 & 3 & 5 \\
\hline $\bar{c}$. circumscissa & 4 & 6 & 5 & $<1$ & $<1$ & $<1$ \\
\hline C. pterocarya & 2 & 1 & 1 & $<1$ & $<1$ & $<1$ \\
\hline P. Iinearis & $<1$ & 1 & $<1$ & $<1$ & 0 & $<1$ \\
\hline D. verna & - & - & - & 8 & 6 & 7 \\
\hline ㅍ. umbe11 atum & - & - & - & 10 & 2 & 7 \\
\hline A. 1ycopsoides & $<1$ & 0 & $<1$ & $<1$ & $<1$ & $<1$ \\
\hline Astragalus & 0 & 1 & $<1$ & - & - & - \\
\hline B. careyana & $<1$ & $<1$ & $<1$ & - & - & - \\
\hline B. dougiasii & 0 & $<1$ & $<1$ & - & - & - \\
\hline ‥ pa11ida & 0 & $<1$ & $<1$ & - & - & - \\
\hline C. terebinthinus & $<1$ & 1 & $<1$ & - & - & - \\
\hline C. macrocarpus & 0 & $<1$ & $<1$ & - & - & - \\
\hline E. asperum & 1 & 0 & $<1$ & - & - & - \\
\hline Eriogonum & $<1$ & $<1$ & $<1$ & 2 & 8 & 5 \\
\hline S. kali & $<1$ & $<1$ & $<1$ & $<1$ & $<1$ & $<1$ \\
\hline$\overline{\mathrm{s}}$. $\overline{\text { altiss imum }}$ & 0 & $<1$ & $<1$ & & & \\
\hline $\bar{M}$. albicaulis & 0 & $<1$ & $<1$ & & & \\
\hline Lomatium & - & - & - & 0 & $<1$ & $<1$ \\
\hline Unknowns & $<1$ & 0 & $<1$ & $<1$ & $<1$ & $<1$ \\
\hline P. longifolia & $\leq 1$ & 4 & $\underline{2}$ & - & - & - \\
\hline Total & $\overline{42}$ & $\overline{47}$ & $\overline{44}$ & $\overline{28}$ & $\overline{23}$ & $\overline{25}$ \\
\hline
\end{tabular}


TABLE 7. Total understory cover (\%) at the B-C Cribs and WyeBarricade habitats with statistical significance.

\begin{tabular}{|c|c|c|c|c|c|c|}
\hline \multirow[b]{2}{*}{ Transect } & \multicolumn{3}{|c|}{ Rep 1} & \multicolumn{3}{|c|}{ Rep 2} \\
\hline & $n$ & $\bar{X} \pm S E$ & C.V. 1 & $n$ & $\bar{X} \pm S E$ & C.V. \\
\hline \multicolumn{7}{|c|}{ B-C Cribs Understory } \\
\hline \multirow[t]{2}{*}{$\begin{array}{l}1 \\
2 \\
3 \\
4\end{array}$} & $\begin{array}{r}50 \\
50 \\
50 \\
50 \\
200\end{array}$ & $\begin{array}{l}33.2 \pm 3.0 \\
36.0 \pm 3.1 \\
47.6 \pm 3.5 \\
38.8 \pm 2.8 \\
38.9 \pm 1.6\end{array}$ & $\begin{array}{l}.65 \\
.61 \\
.53 \\
.50 \\
.58\end{array}$ & $\begin{array}{r}50 \\
50 \\
50 \\
50 \\
200\end{array}$ & $\begin{array}{l}33.7 \pm 2.4 \\
29.6 \pm 2.5 \\
35.3 \pm 2.5 \\
35.2 \pm 3.4 \\
33.4 \pm 1.4\end{array}$ & $\begin{array}{l}.50 \\
.60 \\
.50 \\
.68 \\
.58\end{array}$ \\
\hline & & Rep & +2 & 400 & $36.2 \pm 1.1$ & .59 \\
\hline
\end{tabular}

Wye-Barricade Understory

\begin{tabular}{rrrrrrr}
1 & 50 & $51.1 \pm 4.6$ & .63 & 50 & $52.8 \pm 4.7$ & .62 \\
& $\frac{50}{100}$ & $\frac{63.8 \pm 3.9}{57.8 \pm 3.1}$ & $\frac{.43}{.53}$ & $\frac{50}{100}$ & $\frac{50.3 \pm 4.6}{51.5 \pm 3.3}$ & $\frac{.65}{.63}$ \\
& $\operatorname{Rep~1+Rep~2~}$ & 200 & $54.7 \pm 2.2$ & .58 \\
\hline
\end{tabular}

\section{Statistical Treatment}

\section{$\underline{\text { Treatment }}$}

B-C Cribs site vs. Wye-Barricade Site Replicates within habitats Transects within replicates

\section{Significance}

$P \leq .01 \quad S$

$P \equiv .23$ NS

$P \leq .001 \quad S$

1/Sample size, mean \pm standard error of the mean and coefficient of variation. 
TABLE 8. Tota1 cover (\%) excluding cheatgrass of the understory at the B-C cribs and Wye-Barricade habitats with statistical treatment.

\begin{tabular}{|c|c|c|c|c|c|c|}
\hline \multirow[b]{2}{*}{ Transect } & \multicolumn{3}{|c|}{$\operatorname{Rep} 1$} & \multicolumn{3}{|c|}{ Rep 2} \\
\hline & $n$ & $\bar{X} \pm S E$ & C.V. II & $n$ & $\bar{X} \pm S E$ & C.V. \\
\hline \multirow{3}{*}{$\begin{array}{l}1 \\
2 \\
3 \\
4\end{array}$} & \multicolumn{6}{|c|}{ B-C Cribs Understory } \\
\hline & $\begin{array}{r}50 \\
50 \\
50 \\
50 \\
200\end{array}$ & $\begin{array}{l}20.2 \pm 1.9 \\
20.3 \pm 2.2 \\
21.6 \pm 2.1 \\
22.1 \pm 1.6 \\
21.1 \pm 0.9\end{array}$ & $\begin{array}{l}0.64 \\
0.75 \\
0.69 \\
0.53 \\
0.65\end{array}$ & $\begin{array}{r}50 \\
50 \\
50 \\
50 \\
200\end{array}$ & $\begin{array}{l}23.4 \pm 2.1 \\
20.5 \pm 1.6 \\
22.7 \pm 1.9 \\
15.8 \pm 2.1 \\
20.6 \pm 1.0\end{array}$ & $\begin{array}{l}0.64 \\
0.53 \\
0.59 \\
0.96 \\
0.68\end{array}$ \\
\hline & & $\operatorname{Rep}$ & $+\operatorname{Rep} 2$ & 400 & $20.9 \pm 0.7$ & 0.66 \\
\hline \multirow{3}{*}{$\begin{array}{l}1 \\
2\end{array}$} & \multicolumn{6}{|c|}{ Wye-Barricade Understory } \\
\hline & $\begin{array}{r}50 \\
50 \\
100\end{array}$ & $\begin{array}{l}16.2 \pm 2.1 \\
28.6 \pm 3.1 \\
22.4 \pm 2.0\end{array}$ & $\begin{array}{l}0.91 \\
0.77 \\
0.88\end{array}$ & $\begin{array}{r}50 \\
50 \\
100\end{array}$ & $\begin{array}{r}16.5 \pm 2.5 \\
17.9 \pm 3.2 \\
17.2 \pm 2.0\end{array}$ & $\begin{array}{l}1.05 \\
1.26 \\
1.17\end{array}$ \\
\hline & & Rep & $+\operatorname{Rep} 2$ & 200 & $19.8 \pm 1.4$ & 1.00 \\
\hline
\end{tabular}

\section{Statistical Treatment}

Treatment

B-C Cribs site vs. Wye-Barricade site Replicates within habitats. Transects within replicates $\underline{\text { Significance }}$

$$
\begin{array}{ll}
P \geq .05 & N S \\
P=.19 & N S \\
P \leq .001 & S
\end{array}
$$

1/ Sample size, mean \pm standard error of the mean and coefficient of variation. 
TABLE 9. Cheatgrass cover (\%) at the B-C Cribs and Wye-Barricade habitats with statistical treatment.

Transect

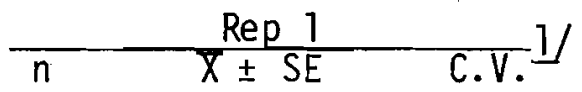

$\operatorname{Rep} 2$

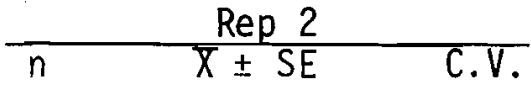

\begin{tabular}{|c|c|c|c|c|c|c|}
\hline & \multicolumn{6}{|c|}{ B-C Cribs Understory } \\
\hline \multirow[t]{2}{*}{$\begin{array}{l}1 \\
2 \\
3 \\
4\end{array}$} & $\begin{array}{r}50 \\
50 \\
50 \\
50 \\
200\end{array}$ & $\begin{array}{l}13.0 \pm 2.1 \\
15.6 \pm 2.3 \\
25.9 \pm 2.2 \\
16.7 \pm 2.3 \\
17.8 \pm 1.3\end{array}$ & $\begin{array}{l}1.12 \\
1.07 \\
0.87 \\
0.98 \\
1.03\end{array}$ & $\begin{array}{r}50 \\
50 \\
50 \\
50 \\
200\end{array}$ & $\begin{array}{r}10.2 \pm 1.4 \\
9.1 \pm 1.7 \\
12.6 \pm 1.9 \\
19.4 \pm 3.0 \\
12.8 \pm 1.1\end{array}$ & $\begin{array}{l}0.99 \\
1.30 \\
1.08 \\
1.08 \\
1.10\end{array}$ \\
\hline & & $\begin{array}{r}\text { Rep } \\
\text { Wy }\end{array}$ & $\begin{array}{l}+\operatorname{Rep} 2 \\
\text { Barricar }\end{array}$ & $\begin{array}{l}400 \\
\text { derst }\end{array}$ & $\begin{array}{l}15.3 \pm 0.9 \\
y\end{array}$ & 1.11 \\
\hline \multirow[t]{5}{*}{$\begin{array}{l}1 \\
2\end{array}$} & $\begin{array}{r}50 \\
50 \\
100\end{array}$ & $\begin{array}{l}35.7 \pm 4.7 \\
35.1 \pm 4.2 \\
35.4 \pm 2.9\end{array}$ & $\begin{array}{l}0.80 \\
0.85 \\
0.82\end{array}$ & $\begin{array}{r}50 \\
50 \\
100\end{array}$ & $\begin{array}{l}36.3 \pm 4.9 \\
32.4 \pm 4.3 \\
34.3 \pm 3.2\end{array}$ & $\begin{array}{l}0.94 \\
0.94 \\
0.93\end{array}$ \\
\hline & & Rep & $+\operatorname{Rep} 2$ & 200 & $34.9 \pm 2.2$ & 0.89 \\
\hline & \multicolumn{6}{|c|}{ Statistical Treatment } \\
\hline & \multicolumn{4}{|c|}{ Treatment } & \multicolumn{2}{|c|}{ Significance } \\
\hline & $\begin{array}{l}\text { B-C } \\
\text { Rep } 1 \\
\text { Tran }\end{array}$ & $\begin{array}{l}\text { ibs site vs } \\
\text { ates within } \\
\text { cts within }\end{array}$ & $\begin{array}{l}\text { ye-Barr } \\
\text { bitats. } \\
\text { licates }\end{array}$ & site & $\begin{array}{l}P \leq . \\
P=. \\
P=.\end{array}$ & $\begin{array}{r}\mathrm{S} \\
\mathrm{NS} \\
\mathrm{S}\end{array}$ \\
\hline
\end{tabular}

1/Sample size, mean \pm standard error of the mean and coefficient of variation. 
The variation associated with the understory parameters measured ranged from 0.58 to 1.11 on both sites, while the shrub parameters measured had a range from 0.28 to 1.10 (Table 10). Bitterbrush and

TABLE 10. Coefficient of variations (C.V.) of various parameters measured at the B-C Cribs and WyeBarricade habitats.

\begin{tabular}{|c|c|c|}
\hline Measurement & B-C Cribs & Wye-Barricade \\
\hline Total understory cover & .59 & .58 \\
\hline Cheatgrass (B. tectorum) & 1.11 & .89 \\
\hline Total cover excluding cheatgrass & .66 & 1.00 \\
\hline Total overstory (shrub) cover ( $L \times W)$ & .90 & .86 \\
\hline Sagebrush (… tridentata) & .91 & 1.10 \\
\hline Bitterbrush (‥ tridentata) & - & .81 \\
\hline Rabbitbrush (‥ nauseosus) & - & .78 \\
\hline Rabbitbrush (‥ viscidiflorus) & .64 & .75 \\
\hline Total shrub height & .33 & .35 \\
\hline Sagebrush (‥ tridentata) & .33 & .38 \\
\hline Bitterbrush (P. tridentata) & - & .28 \\
\hline Rabbitbrush (‥ nauseosus) & - & .31 \\
\hline Rabbitbrush (‥ viscidiflorus) & .20 & .43 \\
\hline
\end{tabular}

rabbitbrush were less variable in canopy cover than sagebrush on both sites. For all parameters measured, heights of shrubs were the least variable for all species. The coefficient of variation ranged between 0.20 to 0.43 on the two habitats.

Frequency of occurrence as outlined by Hyder et al. (1965) was converted to percent frequency and is shown in Table 11 for plants occurring within both habitats. Grasses contributed 41 and $37 \%$ of the total species, while forbs contributed 51 and $56 \%$ on the B-C Cribs and WyeBarricade sites, respectively. A preliminary list of common plants occurring within the habitats is presented in Appendix, Table A-1. 
TABLE 11. Percent of botanical composition of plant species calculated from frequency occurrence at the B-C Cribs and Wye-Barricade habitats.

\begin{tabular}{|c|c|c|c|c|c|c|}
\hline \multirow[b]{2}{*}{ Plant Species } & \multicolumn{3}{|c|}{ B-C Cribs } & \multicolumn{3}{|c|}{ Wye-Barricade } \\
\hline & $\overline{\operatorname{Rep} 1}$ & Rep & Avg. & $\operatorname{Rep}$ & $\operatorname{Rep}$ & Avg. \\
\hline & \multicolumn{3}{|c|}{---- Percent-n- } & \multicolumn{3}{|c|}{---- Percent------ } \\
\hline \multicolumn{7}{|l|}{ Grasses } \\
\hline B. tectorum & 21 & 20 & 20 & 25 & 28 & 26 \\
\hline F. octoflora & 15 & 18 & 17 & $<1$ & 0 & $<1$ \\
\hline $\bar{P} . \overline{\text { sandbergi } i}$ & 7 & 3 & 5 & 10 & 10 & 10 \\
\hline S. & - & - & - & 0 & $<1$ & $<1$ \\
\hline Total & $\overline{43}$ & $\overline{4 T}$ & $\overline{41}$ & $\overline{35}$ & $\overline{38}$ & $\overline{36}$ \\
\hline \multicolumn{7}{|l|}{ Forbs } \\
\hline D. pinnata & 19 & 17 & 18 & $<1$ & 2 & 1 \\
\hline M. gracilis & 16 & 12 & 14 & 14 & 12 & 13 \\
\hline ㄷ. circumscissa & 11 & 10 & 10 & 5 & 3 & 4 \\
\hline $\bar{c} . \overline{\text { pterocarya }}$ & 3 & 2 & 3 & 1 & 3 & 2 \\
\hline P. Tinearis & $<1$ & 3 & 1 & $<1$ & 0 & $<1$ \\
\hline D. verna & - & - & - & 18 & 21 & 19 \\
\hline H. umbe11 atum & - & - & - & 11 & 4 & 8 \\
\hline A. Tycopsoides & $<1$ & 0 & $<1$ & $<1$ & $<1$ & $<1$ \\
\hline Astragalus & 0 & 1 & $<1$ & - & - & - \\
\hline B. careyana & $<1$ & $<1$ & $<1$ & - & - & - \\
\hline B. douglasii & 0 & $<1$ & $<1$ & - & - & - \\
\hline $\bar{C} . \overline{\text { pallida }}$ & 0 & $<1$ & $<1$ & - & - & - \\
\hline c. terebinthinus & $<1$ & $<1$ & $<1$ & - & - & - \\
\hline C. macrocarpus & 0 & $<1$ & $<1$ & - & - & - \\
\hline $\bar{E} . \overline{\text { asperum }}$ & 1 & 0 & $<1$ & - & - & - \\
\hline Eriogonum & $<1$ & 1 & $<1$ & 3 & 6 & 4 \\
\hline S. kali & $<1$ & 1 & $<1$ & $<1$ & $<1$ & $<1$ \\
\hline S. altissimum & 0 & $<1$ & $<1$ & - & - & - \\
\hline M. albicaulis & 0 & $<1$ & $<1$ & - & - & - \\
\hline Eomatium & - & - & - & 0 & $<1$ & $<1$ \\
\hline 0. pallida & $<1$ & $<1$ & $<1$ & - & - & - \\
\hline Unknowns & $<1$ & 0 & $<1$ & 2 & 3 & 2 \\
\hline Total & $\overline{52}$ & $\overline{49}$ & $\overline{51}$ & $\overline{56}$ & $\overline{56}$ & $\overline{56}$ \\
\hline \multicolumn{7}{|l|}{ Shrubs } \\
\hline P. tridentata & - & - & - & 7 & 5 & 6 \\
\hline A. Eridentata & 5 & 9 & 8 & 2 & 1 & 1 \\
\hline C. $\overline{\text { nauseosus }}$ & 0 & 0 & 0 & $<1$ & $<1$ & $<1$ \\
\hline C. viscidiflorus & $<1$ & 0 & $<1$ & $<1$ & $<1$ & $<1$ \\
\hline P. Tongifolia & $<1$ & 1 & $<1$ & - & - & - \\
\hline Total & 5 & $\overline{10}$ & 8 & $\overline{9}$ & $\overline{6}$ & 7 \\
\hline
\end{tabular}


BNWL-1931

\section{FECAL PELLET ANALYSES}

Recent Pellets

Nine plant species were identified in recent pellets at the $B-C$ Cribs Control zone as compared to 11 species at the Wye-Barricade (Table 12). Yarrow, Achillea millifolium, was the most often encountered species, comprising 29 and $21 \%$ of the identifiable plant fragments in the B-C Cribs Control zone and Wye-Barricade samples, respectively. The plant species identified in pellets from the B-C Cribs sites listed in decreasing order of abundance are: yarrow > turpentine cymopterus > tumble mustard > erigeron > needle-and-thread > matted cryptantha > rabbitbrush > sagebrush. The important species of the Wye-Barricade site listed in decreasing order of abundance are: yarrow > turpentine cymopterus > erigeron > needle-and-thread > rabbitbrush > Sandberg bluegrass > scurf pea. Forbs provided the mainstay of the hare diet. Eightyone percent of identified plant parts at the B-C Cribs were forbs as compared to $69 \%$ at the Wye-Barricade (Figure 2 ).

When a plant species is scarce in the field but shows up frequently in fecal pellets, it is assumed that this particular plant is actively sought by the foraging animal. The data 1 isted in Table 13 show that yarrow is a preferred forage, as well as turpentine cymopterus, needleand-thread, erigeron, and tumble mustard. On the other hand, cheatgrass, abundant in both study sites, did not appear in the fecal pellets at all, thereby indicating that hares selectively avoided cheatgrass. Russian thistle, Salsola kali, was sparsely represented in both study areas and did not appear in the pellet analyses. A list of plants identified in fecal pellets is presented in Appendix, Table A-2.

Indices of similarity between diets and habitats are comparisons that show a relationship between food items and the same items observed in the habitat. A similarity index of 100 indicates that hares are foraging upon plants in the same proportion as they actually occur in the field. A small index number indicates that the hare's diet preference is less compatible with the vegetation in the field. Sandberg bluegrass, matted cryptantha, and sagebrush had high similarities while other species in the diet showed low similarity indices (Table 14). 
TABLE 12. Plant species identified in recent hare pellets collected at B-C Cribs and Wye-Barricade habitats with percent frequency.

\begin{tabular}{lcc}
\hline Plant Species & B-C Cribs & Wye-Barricade \\
\hline Grasses & & \\
Stipa & comata & 11 \\
Poa sandbergii & 0 & 10 \\
Forbs & & \\
Achillea mil1ifolium & 29 & 21 \\
Cymopterus terebinthinus & 17 & 19 \\
Sisymbrium $\frac{\text { tiltissimum }}{\text { Erigeron sp. }}$ & 14 & 7 \\
Balsamorhiza careyana & 13 & 16 \\
Psoralea lanceolata & 0 & 4 \\
Cryptantha circumscissa & 1 & $<1$ \\
C. pterocarya & 6 & $<1$ \\
Unknown & 0 & 1 \\
Shrubs & 1 & 10 \\
Chrysothamnus nauseosus & & 0 \\
Artemisia tridentata & 4 & 4 \\
\hline
\end{tabular}


BINWL-193T

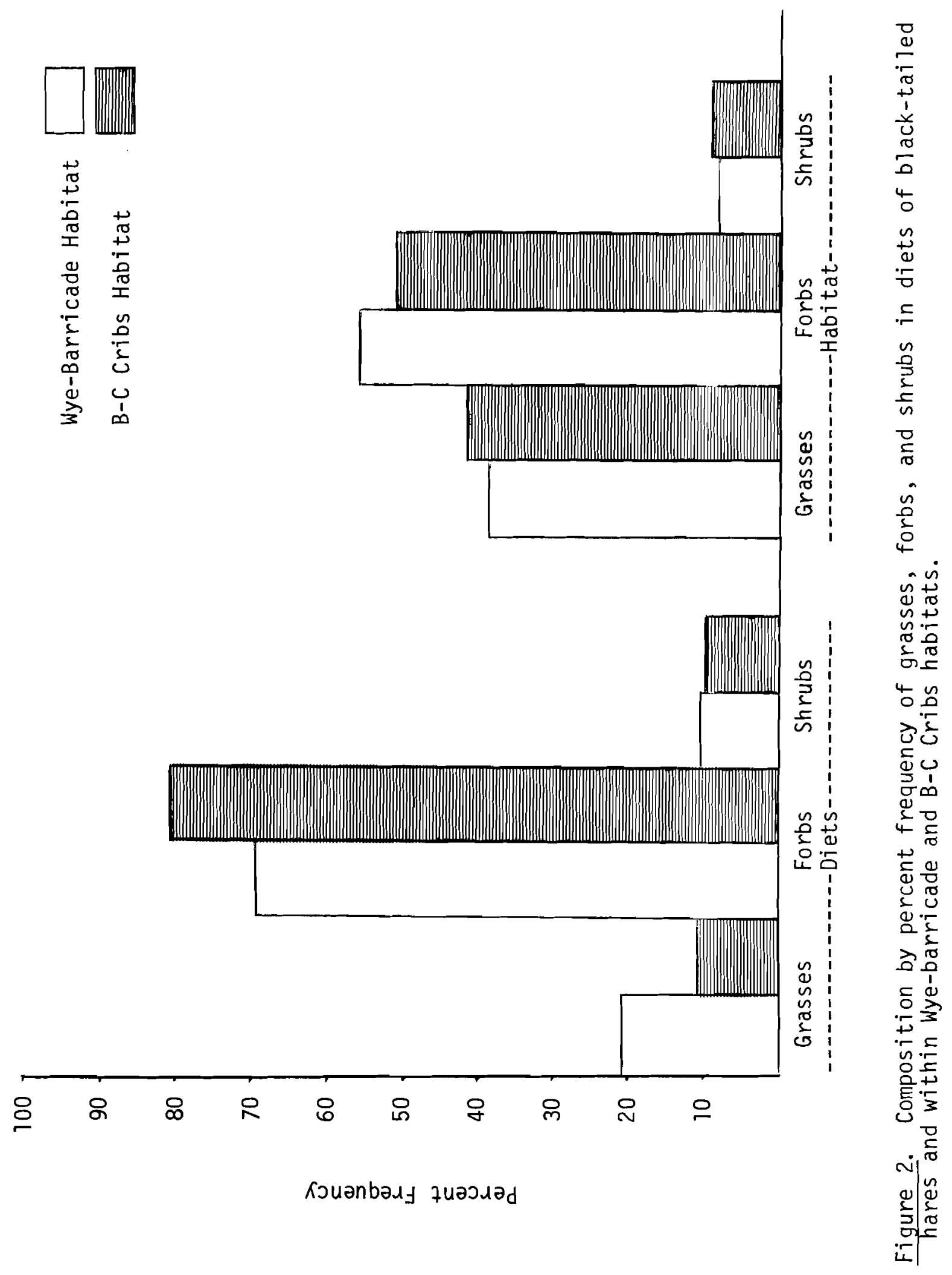


TABLE 13. Preference indices for the most important plants found in the diet of the black-tailed hare from recent and all pellets combined at the B-C Cribs and Wye-Barricade habitats.

\begin{tabular}{|c|c|c|c|c|}
\hline \multirow[b]{2}{*}{ Plant Taxa } & \multicolumn{2}{|c|}{ B-C Cribs } & \multicolumn{2}{|c|}{ Wye-Barricade } \\
\hline & Recent & A11 Pellets & Recent & A11 Pellets \\
\hline \multicolumn{5}{|l|}{ Grasses } \\
\hline S. comata & 110.0 & 140.0 & 110.0 & 130.0 \\
\hline ㅁ. sandbergii & - & $<0.1$ & 1.0 & 0.8 \\
\hline \multicolumn{5}{|l|}{ Forbs } \\
\hline A. millifolium & 290.0 & 270.0 & 270.0 & 220.0 \\
\hline $\bar{c}$ terebinthinus & 170.0 & 160.0 & 190.0 & 180.0 \\
\hline Erigeron sp. & 130.0 & 140.0 & 160.0 & 170.0 \\
\hline S. altissimum & 140.0 & 130.0 & 70.0 & 70.0 \\
\hline c. circumscissa & 0.6 & 0.4 & $<0.1$ & 0.2 \\
\hline P. Tanceolata & 10.0 & 1.0 & 40.0 & 40.0 \\
\hline B. careyana & - & - & $<0.1$ & 1.0 \\
\hline C. pterocarya & - & - & $<0.1$ & $<0.1$ \\
\hline D. verna & - & $<0.1$ & - & - \\
\hline \multicolumn{5}{|l|}{ Shrubs } \\
\hline A. tridentata & 0.5 & 0.5 & - & - \\
\hline ‥ $\overline{\text { nauseosus }}$ & 40.0 & 50.0 & 100.0 & 90.0 \\
\hline
\end{tabular}


TABLE 14. Kulczynski's index of similarity (SI) comparing plant frequency found in recent pellets with plant frequency at the B-C Cribs and Wye-Barricade habitats.

\begin{tabular}{|c|c|c|}
\hline Plant Taxa & $\begin{aligned} \text { B-C } & \text { Cribs } \\
\% & \text { SI }\end{aligned}$ & $\begin{array}{c}\text { Wye-Barricade } \\
\% \text { SI }\end{array}$ \\
\hline \multicolumn{3}{|l|}{ Grasses } \\
\hline$\underline{\text { P. }} \cdot \underline{\text { s sandbergi }} \cdot \underline{\text { comata }}$ & $\overline{0 .}$ & $\begin{array}{r}100.0 \\
0.2\end{array}$ \\
\hline \multicolumn{3}{|l|}{ Forbs } \\
\hline C. circumscissa & 75.0 & 0.5 \\
\hline $\bar{c} . \overline{\text { pterocarya }}$ & - & 1.0 \\
\hline B. careyana & - & $\star$ \\
\hline c. terebinthinus & 0.1 & 0.1 \\
\hline s. altissimum & 0.1 & 0.3 \\
\hline Erigeron sp. & 0.1 & 0.1 \\
\hline A. mi11ifolium & 0.1 & 0.1 \\
\hline ‥ $\overline{\text { lanceolata }}$ & 1.9 & 0.5 \\
\hline \multicolumn{3}{|l|}{ Shrubs } \\
\hline A. tridentata & 66.6 & - \\
\hline ‥ nauseosus & 0.5 & 0.2 \\
\hline
\end{tabular}

*Present in trace amounts. 
BNWL-1931

Comparison of the mean indices of Kulczynski's index of similarity for new, old, and aged pellets collected at the B-C Cribs Control site showed similarities ranging from 83 to $92 \%$ (Table 15). Within the WyeBarricade habitat, the similarity between new and old-aged pellets was $78 \%$. The mean index of similarity when comparing the two sites was $36 \%$. This indicates that the two sites were not similar in species composition. The average index of similarity between new pellets of both sites was $49 \%$. This suggests that there are some differences in hare diets between the two habitats.

Combined Pellet Classes

The average percent frequency of plant species occurring in recent, old, aged, and old-aged pellets were similar. Grasses were most variable, having an average frequency of $16 \%$ and ranging between 11 and $22 \%$ of the total (Table 16). Forbs were less variable among the different age groups of pellets having an average of $73 \%$ and ranging between 67 and $80 \%$. Shrubs were the least variable among the different classes of pe1lets, averaging about 9\%. A11 major and important food items were present in approximately the same percent frequency for all groups of pellets (Tables 17 and 18). The less important species, making up less than $5 \%$ of the total frequency, varied among the classes. This may be due to the hare's seeking out these plants one year but not the next, or to seasonal changes in plant abundance. These results indicate that the black-tailed hare has selected the major plant species in approximately the same proportion in all age classes of pellets over a period of years.

A comparison of all pellet classes within the B-C Cribs and WyeBarricade habitats shows grasses to comprise 14 and $21 \%$ of the diet, while forbs made up 74 and $70 \%$ in the respective habitats (Table 19). Occurrence of shrub parts in the pellets was approximately the same at both sites and made up $9 \%$ of the composition. Comparison of both habitats shows a significant difference in amounts of Sandberg bluegrass. Sandberg bluegrass made up approximately $8 \%$ of the diet in the WyeBarricade habitat and was presentiy only in trace amounts in pellets from the B-C Cribs habitat. Although sagebrush did not occur in the 
TABLE 15. Mean $(X)$ and standard errors (SE) of Kulczynski's index of similarity $(\%)$ comparina the diets and feeding habits of black-tailed hares and age class of pellets.

\begin{tabular}{lr}
\hline \multicolumn{1}{c}{ Comparisons } & $\bar{X} \pm S E$ \\
\hline B-C Cribs habitat & \\
Recent pellets vs. old pellets & $84 \pm 8$ \\
Recent pellets vs. aged pellets & $83 \pm 3$ \\
Old pellets vs. aged pellets & $92 \pm 1$ \\
Recent pellets vs. old + aged pellets & $83 \pm 3$ \\
& \\
Wye-Barricade habitat & $78 \pm 1$ \\
Recent pellets vs. old + aged pellets & $36 \pm 1$ \\
B-C Cribs habitat vs. Wye-Barricade habitat \\
Recent pellets (B-C Cribs habitat) vs. recent pellets \\
(Wye-barricade habitat)
\end{tabular}


TABLE 16. Average percent frequency of plant fragments occurring in recent, old, aqed, and old-aged black-tailed hare pellets within the B-C Cribs (B) and Wye-Barricade (W) habitats.

\begin{tabular}{|c|c|c|c|c|c|c|}
\hline Plant Taxa & \multicolumn{2}{|c|}{ Recent } & $\frac{01 \mathrm{~d}}{\mathrm{~B}}$ & $\frac{\text { Aged }}{B}$ & \multicolumn{2}{|c|}{$01 d+$ Aged } \\
\hline \multicolumn{7}{|l|}{ Grasses } \\
\hline$\underline{\text { S. }}$ comata & 11 & 11 & 12 & 19 & 15 & 14 \\
\hline$\overline{\underline{p}} . \overline{\text { sandbergi }}$ & - & 10 & $\leq 1$ & - & $<1$ & 5 \\
\hline Tota 1 & $\overline{11}$ & $\overline{22}$ & $\overline{12}$ & $\overline{19}$ & $\frac{1}{15}$ & 19 \\
\hline \multicolumn{7}{|l|}{ Forbs } \\
\hline A. millifolium & 29 & 21 & 26 & 27 & 26 & 23 \\
\hline C. terebinthinus & 17 & 19 & 16 & 16 & 16 & 18 \\
\hline Erigeron sp. & 13 & 16 & 15 & 13 & 14 & 18 \\
\hline S. altissimum & 14 & 7 & 13 & 12 & 12 & 7 \\
\hline c. circumscissa & 6 & $<1$ & 7 & $<1$ & 4 & 3 \\
\hline$\overline{\mathrm{P}} . \overline{\text { lanceolata }}$ & 1 & 4 & $<1$ & - & $<1$ & 3 \\
\hline$\underline{\bar{B}}$. careyana & - & $<1$ & - & - & - & - \\
\hline$\overline{\bar{c}}$. $\overline{\text { pterocarya }}$ & - & $<1$ & - & - & - & $<1$ \\
\hline D. verna & - & - & $<1$ & - & $<1$ & \\
\hline Total & $\overline{80}$ & $\overline{67}$ & $\overline{77}$ & $\overline{69}$ & $\overline{72}$ & $\overline{72}$ \\
\hline \multicolumn{7}{|l|}{ Shrubs } \\
\hline A. tridentata & 4 & - & 5 & 3 & 4 & - \\
\hline $\bar{c} . \overline{\text { nauseosus }}$ & 4 & 10 & 5 & 6 & 5 & 9 \\
\hline Total & 8 & $\overline{10}$ & $\overline{10}$ & 9 & $\overline{9}$ & $\overline{9}$ \\
\hline Unknowns & 1 & 1 & $<1$ & $<1$ & $<1$ & $<1$ \\
\hline
\end{tabular}


TABLE 17. Percent frequency of plant species occurring in recent, old, and aged blacktailed hare pellets at the B-C Cribs habitat.

\begin{tabular}{|c|c|c|c|c|c|c|c|c|c|}
\hline \multirow[b]{2}{*}{ Plant Taxa } & \multicolumn{3}{|c|}{ Recent } & \multicolumn{3}{|c|}{ 01d } & \multicolumn{3}{|c|}{ Aged } \\
\hline & $\overline{\operatorname{Rep} 1}$ & $\operatorname{Rep} 2$ & Avg. & $\overline{\operatorname{Rep} 1}$ & $\operatorname{Rep} 2$ & Avg. & $\overline{\operatorname{Rep} 1}$ & $\operatorname{Rep} 2$ & Avg. \\
\hline \multicolumn{10}{|l|}{ Grasses } \\
\hline S. comata & 4 & 17 & 11 & 13 & 11 & 12 & 22 & 17 & 19 \\
\hline$\underline{P} \cdot$ sandbergii & - & $\overline{-}$ & $\overline{\overline{71}}$ & $\frac{1}{1 \pi}$ & $\frac{0}{77}$ & $\leq 1$ & $\overline{-}$ & $\overline{-}$ & $\overline{\overline{10}}$ \\
\hline Total & $\overline{4}$ & 77 & 71 & $\overline{14}$ & $\overline{11}$ & $\overline{12}$ & $\overline{22}$ & 17 & $\overline{19}$ \\
\hline \multicolumn{10}{|l|}{ Forbs } \\
\hline A. millifolium & 28 & 30 & 29 & 24 & 28 & 26 & 26 & 28 & 27 \\
\hline $\bar{c}$. terebinthinus & 15 & 18 & 17 & 17 & 16 & 16 & 16 & 16 & 16 \\
\hline Erigeron sp. & 13 & 14 & 13 & 16 & 13 & 15 & 14 & 13 & 13 \\
\hline s. altissimum & 19 & 10 & 14 & 10 & 16 & 13 & 14 & 11 & 12 \\
\hline $\bar{c} . \overline{c i r c u m s c i s s a}$ & 7 & 4 & 6 & 9 & 4 & 7 & 1 & 1 & 1 \\
\hline$\overline{\underline{P}} . \overline{\text { 1anceolata }}$ & 2 & 0 & 1 & $<1$ & 1 & $<1$ & - & - & - \\
\hline D. verna & $=$ & - & - & $<1$ & 0 & $<1$ & & & \\
\hline Total & $\overline{84}$ & $\overline{76}$ & $\overline{80}$ & $\overline{76}$ & $\overline{78}$ & $\overline{77}$ & $\overline{71}$ & $\overline{69}$ & $\overline{69}$ \\
\hline \multicolumn{10}{|l|}{ Shrubs } \\
\hline A. tridentata & 3 & 4 & 4 & 5 & 3 & 5 & 2 & 5 & 3 \\
\hline ㄷ. nauseosus & 7 & 2 & 4 & 3 & 6 & $\frac{5}{2}$ & $\frac{5}{7}$ & $\frac{8}{1-12}$ & 6 \\
\hline Total & 7 & 6 & 8 & $\overline{8}$ & 9 & $\overline{10}$ & 7 & $\overline{13}$ & $\overline{9}$ \\
\hline Unknowns & 2 & 1 & 1 & $<1$ & $<1$ & $<1$ & $<1$ & 1 & $<1$ \\
\hline
\end{tabular}


TABLE 18. Percent frequency of plant species occurring in recent, old, and aged black-tailed hare pellets in the wyeBarricade habitat.

\begin{tabular}{|c|c|c|c|c|c|c|}
\hline \multirow[b]{2}{*}{ Plant Taxa } & \multicolumn{3}{|c|}{ Recent } & \multicolumn{3}{|c|}{$01 d+$ Aged } \\
\hline & $\overline{\operatorname{Rep} 1}$ & $\operatorname{Rep} 2$ & Avg. & $\overline{R e p ~} 1$ & $\operatorname{Rep} 2$ & Avg. \\
\hline \multicolumn{7}{|l|}{ Grasses } \\
\hline S. comata & 6 & 17 & 11 & 13 & 14 & 14 \\
\hline P. Sandbergi i & 11 & $\frac{10}{10}$ & 10 & $\underline{6}$ & 4 & 5 \\
\hline Total & & 27 & & & 18 & $\overline{19}$ \\
\hline \multicolumn{7}{|l|}{ Forbs } \\
\hline A. millifolium & 24 & 18 & 21 & 22 & 24 & 23 \\
\hline C. terebinthinus & 24 & 13 & 19 & 18 & 18 & 18 \\
\hline Erigeron sp. & 17 & 15 & 16 & 18 & 18 & 18 \\
\hline s. altissimum & 3 & 11 & 7 & 5 & 8 & 7 \\
\hline C. circumscissa & $<1$ & 0 & $<1$ & 4 & 2 & 3 \\
\hline P. lanceolata & 4 & 4 & 4 & 3 & 3 & 3 \\
\hline B. careyana & $<1$ & 0 & $<1$ & - & - & - \\
\hline C. pterocarya & 1 & 0 & $\leq 1$ & $\leq 1$ & 0 & $\leq 1$ \\
\hline Total & $\overline{73}$ & $\overline{61}$ & $\overline{68}$ & $\overline{70}$ & $\overline{73}$ & $\overline{72}$ \\
\hline \multicolumn{7}{|l|}{ Shrubs } \\
\hline A. tridentata & - & - & - & - & - & - \\
\hline C. nauseosus & 7 & 11 & 10 & 11 & 8 & 9 \\
\hline Total & 7 & $\overline{11}$ & $\overline{10}$ & $\overline{11}$ & $\overline{8}$ & $\overline{9}$ \\
\hline Unknowns & 1 & 1 & 1 & $<1$ & $<1$ & $<1$ \\
\hline
\end{tabular}


TABLE 19. Average percent frequency of plant fragments occurring in all classes of pellets collected from the B-C Cribs and Wye-Barricade habitats.

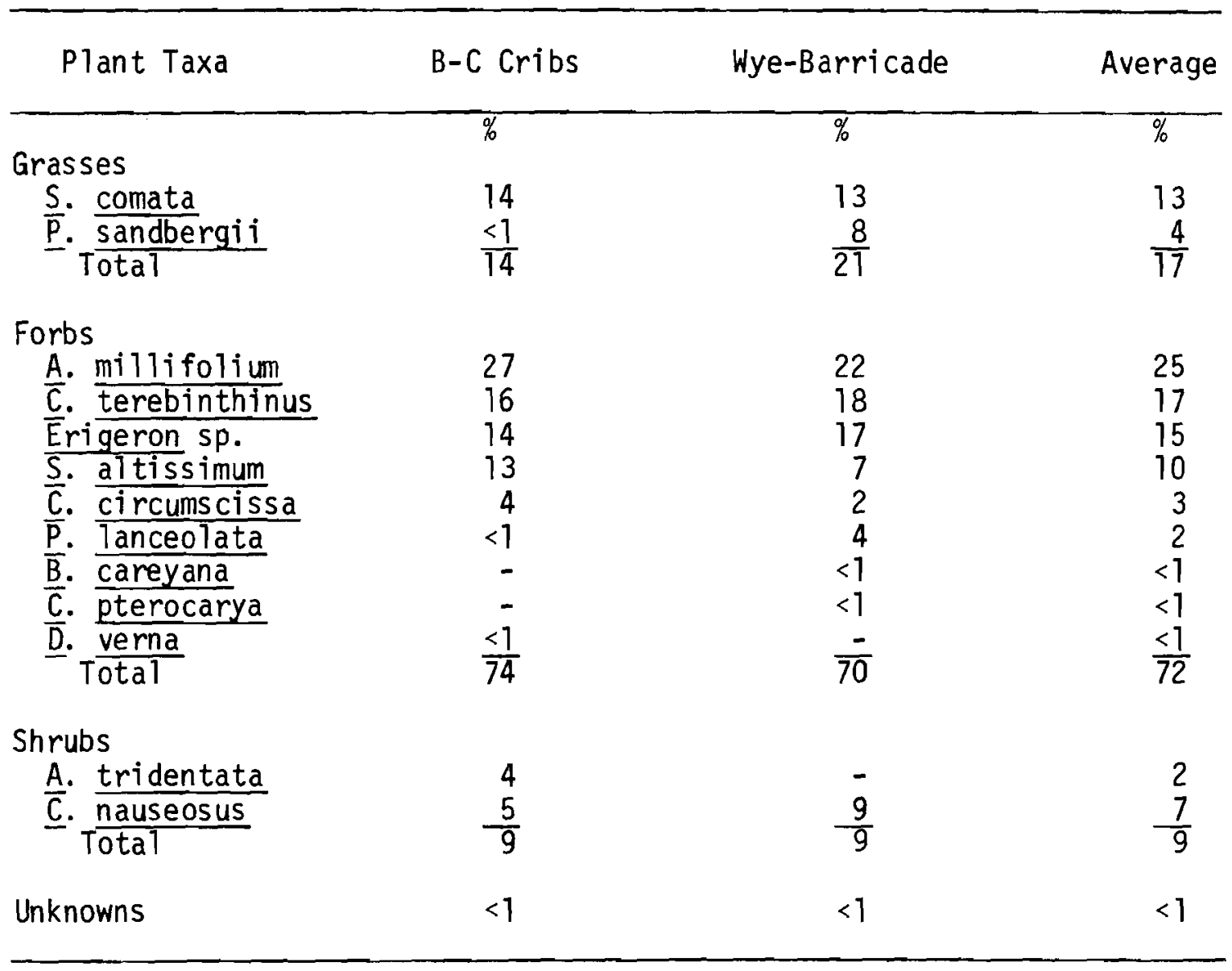


pellets collected from the Wye-Barricade site, it made up $4 \%$ of the dietary composition of pellets from the B-C Cribs site.

The average dietary composition at both sites consisted of $17 \%$ grasses, $72 \%$ forbs, and 9\% shrubs (Table 19).

Plant Parts in Radioactive and Non-Radioactive Pellets

In an investigation at the B-C Cribs Controlled area, pellets were segregated into new pellets, old pellets, and radioactive pellets* (all old) (Table 20). Fourteen plant species were identified in these pellets. Three of these were grasses, eight were forbs, and three shrubs (Table 20). Five species were sparingly represented, i.e., $<1 \%$ of identifiable plant parts. These were squirreltail, balsamroot, blazing star, scurf pea, and big sagebrush. Ninety-two percent of the identifiable plant parts were contributed by the remaining nine species. The most important plants were turpentine cymopterus, erigeron, rabbitbrush, Sandberg bluegrass, and common yarrow. Only eight species of plants were identified in the radioactive pellets, but the number of pellets examined was 1 imited.

Radioactive pellets were at least 10 years old because trenches which were burrowed into by native animals and exposing the salts to the hares were filled and sealed with asphalt in 1964 (O'Farrell and Gilbert, 1975; Maxfield, 1974). The dispersal of radionuclides in fecal pellets is believed to have resulted from hares consuming radioactive salts. The data indicate that hares relied upon the same plants for forage 10 years ago as they do at the present time.

*Determined by hand-held survey meter. 
TABLE 20. Plant species and composition (\%) in the pellets of black-tailed hares collected within the B-C cribs Controlled zone

\begin{tabular}{|c|c|c|c|c|}
\hline Plant Species & New ${ }^{1}$ & $01 d^{2}$ & Radioactive ${ }^{3}$ & Avg. ${ }^{4}$ \\
\hline $\begin{array}{l}\text { Grasses } \\
\text { Poa sandbergii } \\
\frac{\text { Sitanion hystrix }}{\text { Stipa comata }}\end{array}$ & $\begin{array}{l}12 \\
<1\end{array}$ & $\begin{array}{l}15 \\
<1\end{array}$ & $\begin{array}{l}2 \\
9\end{array}$ & $\begin{array}{r}11 \\
<1 \\
2\end{array}$ \\
\hline $\begin{array}{l}\text { Forbs } \\
\text { Achillea mi } 11 \text { ifolium } \\
\frac{\text { Balsamorhiza careyana }}{\text { Cryptantha }} \frac{\text { circumscissa }}{\text { Cerebinthinus }} \\
\text { Cymopterus } \\
\text { Erigeron species } \\
\frac{\text { Mentzelia }}{\text { Psoralea }} \frac{\text { laevicaulis }}{\text { Sisceolata }} \\
\text { Sisybrium altissimum }\end{array}$ & $\begin{array}{l}5 \\
2 \\
11 \\
22 \\
15 \\
<1 \\
<1 \\
<1\end{array}$ & $\begin{array}{l}17 \\
<1 \\
19 \\
31 \\
<1 \\
<1\end{array}$ & $\begin{array}{l}16 \\
13 \\
14\end{array}$ & $\begin{array}{r}11 \\
<1 \\
5 \\
20 \\
20 \\
<1 \\
<1 \\
3\end{array}$ \\
\hline $\begin{array}{l}\text { Shrubs } \\
\text { Artemisia tridentata } \\
\frac{\text { Chrysothamnus nauseosus }}{\text { Grayia spinosa }}\end{array}$ & $\begin{array}{r}2 \\
13 \\
13\end{array}$ & 13 & $\begin{array}{r}2 \\
21\end{array}$ & $\begin{array}{r}1 \\
15 \\
5\end{array}$ \\
\hline Unknown & & & 12 & 3 \\
\hline $\begin{array}{l}{ }^{1} N=16 \text { pellets } \\
{ }^{2} N=14 \text { pellets } \\
{ }^{3} N=9 \text { pellets } \\
{ }^{4} N=39 \text { pellets }\end{array}$ & & & & \\
\hline
\end{tabular}




\section{DISCUSSION}

Plant selectivity by the black-tailed hare has been reported by several investigators. Selectivity is influenced by phenological stages of plant development within growing seasons (Hayden, 1966; Sparks, 1968; Hansen and Flinders, 1969; Flinders, 1971; and Flinders and Hansen, 1972). Hayden (1966) and Sparks (1968) showed that grasses were the important food items during early spring and summer, with forbs important during the summer, while shrubs were more important in winter. Hares exhibited a preference for green vegetation at a11 four seasons of the year. It has been suggested by Hayden (1966) and Sparks (1968) that green, succulent $p l$ ants are preferred by hares to help maintain internal water balance. Selectivity may also be influenced by the amount of available energy stored in certain plants (Church et al., 1972).

It is important to point out that cheatgrass (Bromus tectorum) and Russian thistle (Salsola kali) were not identified in the pellets of the black-tailed hare on the Hanford Reservation. When these two plant species do occur in hare pellets, they are generally found in only trace amounts (Sparks, 1968; Hansen and Flinders, 1969; Flinders and Hansen, 1972).

Stewart and Hul1 (1969) state that hares strongly influence the development and maintenance of cheatgrass cover. Hares feeding heavily on perennial grasses prevented them from invading cheatgrass communities and sometimes even destroyed reseeded grass without eating cheatgrass. On areas that purposefully excluded hares, perennial grasses increased as rapidly as cheatgrass, but these disappeared when grazed by hares and cattle. Hares sometimes girdle main stems of sagebrush and rabbitbrush, which may destroy the plant. Seeds may be transported internally by the hare from area to area, and germination of seeds with durable seed coats may be enhanced after passing through the digestive tract, thus contributing to dispersal and invasion of certain plant species. 
Hansen and Flinders (1969) state that on rangelands deteriorated by livestock grazing, hares exerted forces favoring plant succession toward the climax stage. However, when ranges have deteriorated to a point where forbs ("weedy species") are abundant, then the influence by hares would be toward further deterioration.

Forage consumption by hares averaged $105 \mathrm{~g} /$ day but reached values as high as $260 \mathrm{~g} /$ day in Curlew Valley, Utah, which is similar to the sagebrush habitats on the Hanford Reservation (Currie and Goodwin, 1966). Controlled feeding tests using mixtures of alfalfa and rolled barley showed that black-tailed hares consumed on the average $104 \pm 4.5 \mathrm{~g} / \mathrm{day}$ (Hansen and Flinders, 1969).

Hares that are deprived of salt develop a craving and may resort to licking dirt or other mineral elements (Hansen and Flinders, 1969; and Siegmund, 1969). Pellets collected at Hanford contained fair amounts of soil, suggesting a salt deficiency in the diet.

Natural control of hare populations depends upon the food supply, predation, and disease. Predators of the hare on the Hanford Reservation include the coyote, golden eagle, great-horned ow1, and hawks.

In waste burial zones, man-induced population control of hares may include one or several possibilities: (1) poisoning, (2) trapping, (3) shooting, (4) exclusion, and (5) habitat modification (Vallentine, 1974). Trapping, shooting, and exclusion by fencing may be useful in small areas. Poisoning would only be recommended in extreme cases. Habitat modification may be the most useful long-term control of hares in waste burial zones. Such modifications may change plant species composition to eliminate essential food and cover. Plants that are not consumed by the hare may be considered for the habitat modification. Shrub cover may be removed, since black-tailed hares do not prefer the more open areas (Flinders, 1971; Flinders and Hansen, 1973). However, the welfare of other animals needs to be considered before making such modifications of the habitat. 
BNWL-1931

\section{SUMMARY}

Sixteen species of plants were identified in the fecal pellets of the biack-tailed hare in two habitats-sagebrush-bitterbrush/Sandberg bluegrass and sagebrush/Sandberg bluegrass. Microscopic analys is of plant fragments indicated that yarrow (Achillea millifolium) was the most common food in the diet, making up $25 \%$ of the overall diet. Other plants in the diet listed in order of importance were turpentine cymopterus, erigeron sp., needle-and-thread, and tumble mustard. These five species made up approximately $80 \%$ of the tota 1 diet. Forbs were more important than grasses, and grasses were more important than shrubs. Cheatgrass, the dominant herbaceous species, and Russian thistle, a common forb, did not occur in fecal pellets. The most important plants found in radioactive pellets were turpentine cympoterus, erigeron, rabbitbrush, Sandberg bluegrass, and common yarrow. These five species make up $77 \%$ of the total diet. Eight species of plants were identified in the radioactive pellets, but the number of pellets examined was limited. 
BNWL- 1931

\section{REFERENCES}

Baumgartner, L. L., and A. C. Martin. 1939. Plant histology as an aid in squirrel food habit studies. J. Wild1. Manage. 3:266-268.

Church, D. C. 1972. Digestive Physiology and Nutrition, Vol.3. Oregon State University, Corvallis, Oregon. $349 \mathrm{pp}$.

Currie, P. 0. and D. L. Goodwin. 1966. Consumption of forage by blacktailed jacks on salt-desert ranges of Utah. J. Wildl. Manage. 30:304-311.

Curtis, J. T. and R. P. McIntosh. 1950. The inter-relations of certain analytic and synthetic phytosociological characters. Ecology 31: 434-455.

Daubenmire, R. F. 1959. A canopy-coverage method of vegetation analysis. Northwest Sci. 33:43-64.

Flinders, J. T. 1971. Diets and Feeding Habits of Jackrabbits Within a Shortgrass Ecosystem. Ph.D. Thesis, Colorado State University. $75 \mathrm{pp}$.

Flinders, J. T. and R. M. Hansen. 1972. Diets and Habits of Jackrabbits in Northeastern Colorado. Range Sci. Dept., Ser. 1. 29 pp.

Hansen, R. M. and J. T. Flinders. 1969. Food Habits of North American Hares. Colo. State Univ., Range Sci. Dept., Sci. Ser. 1. 17 pp. Hayden, P. 1966. Food habits of black-tailed jackrabbits in southern Nevada. J. Mamma 1. 47:42-46.

Hyder, D. N., R. E. Bement. E. E. Remmenga, and C. Terwilliger, Jr. 1965. Frequency sampling of blue grama range. J. Range Manage. 18: $90-94$.

Krueger, W. C. 1972. Evaluating animal forage preference. J. Range. Manage. 25:471-475.

Maxfield, H. L. 1974. Prel iminary Safety Analys is of Alternate Proposals for B-C Cribs Controlled Area Action, ARH-3088, Atlantic Richfield Hanford Company, Richland, WA.

Miller, R. G., Jr. 1966. Simultaneous Statistical Influence. McGrawHill Book Co. 272 pp.

0'Farre11, T. P. and R. 0. Gilbert. 1975. Transport of radioactive materials by jackrabbits on the Hanford Reservation. Health Phys. 29:9-15. 
Oosting, H. J. 1956. The Study of Plant Communities. W. H. Freeman and Co., San Francisco, CA. 440 pp.

Siegmund, 0. H. 1967. The Merck Veterinary Manual. Merck and Co., Inc. Rahway, NJ. $1685 \mathrm{pp}$.

Snedecor, G. W. and W. G. Cochran. 1967. Statistical Methods. Iowa State Univ. Press, Ames. $593 \mathrm{pp}$.

Sparks, D. R. 1968. Diets of black-tailed jackrabbits on sandhil1 rangelands in Colorado. J. Range Manage. 21:203-208.

Sparks, D. R. and J. C. Malechek. 1968. Estimating percentage dry weights in diets using a microscopic technique. J. Range Manage. 21:264-265.

Stewart, G. and A. C. Hul1. 1949. Cheatgrass, Bromus tectorum L. -an ecological intruder in southern Idaho. Ecology 30:58-74.

Vallentine, J.F. 1974. Range Developments and Improvements. Brigham Young Univ. Press, Provo, UT. $516 \mathrm{pp}$. 
BNWL-1931

APPENDIX A 
TABLE A-1. List of p1ant species from the B-C Cribs and Wye-Barricade habitat.

Corrmon Name

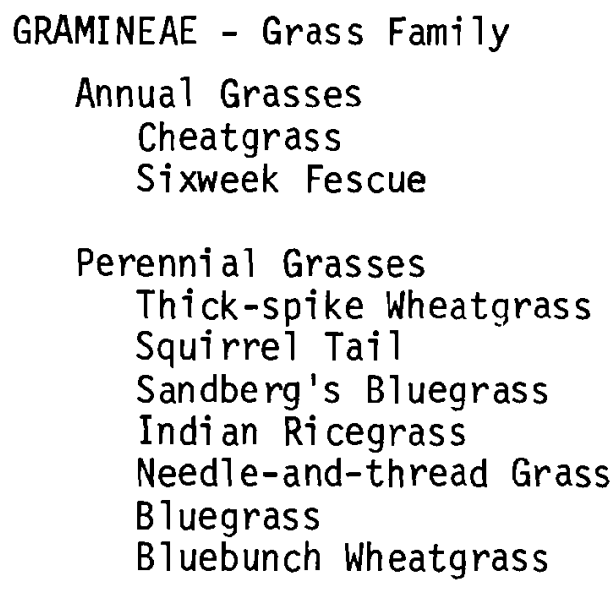

Scientific Name

Bromus tectorum

Festuca octoflora

Agropyron dasytachyum

Sitanion hystrix

Poa sandbergi $i$

Oryzops is hymenoides

Stipa comata

Poa canbyi

Agropyron spicatum

Brodiaea douglasii

Calochort us macrocarpus

Comandra pallida

Eriogonum $s p$.

Salsola kali

Grayia spinosa

Descurainia pinnata

Erysimum asperum

Sisymbrium altissimum Draba verna

Purshia tridentata

Astragalus sp.

Lupinus $\mathrm{sp}$.

Psoralea lanceolata

Erodium cicutarium

Mentzelia albicaulis 
Cormon Name

ONAGRACEAE - Evening Primrose Family

Evening Primrose

UMBELLIFERAE - Parsley Family

Turpentine Cymopterus

POLEMONIACEAE - Ph1ox Family

Long-leaved Phlox

Micros teris

HYDROPHYLLACEAE - Waterleaf Family

Narrow-leaved Phacelis

BORAGINACEAE - Borage Family

Fidd Teneck

Matted Cryptantha

Wing-nut Cryptantha

SCROPHULARIACEAE - Figwort Family

Sharp-leaved Penstemon

COMPOSITAE - Sunflower Family

Big Sagebrush

Rabbi tbrush

Rabbitbrush

Bursage

Balsamroot

Yarrow

Aster species

Erigeron species
Scientific Name

Oenothera pal1ida

Cymopterus terebinthinus

Phlox longifolia

Microsteris gracilis

Phacelis linearis

Amsinckia lycopsoides

Cryptantha circumscissa

Cryptantha pterocarya

Penstemon acuminatus

Artemisia tridentata Chrysothamnus nauseosus

Chrysothamnus viscidiflorus

Franseria acanthicarpa

Balsamorhiza careyana

Achillea mi11 ifolium

Aster sp.

Erigeron $\mathrm{sp}$. 
TABLE A-2. Plant species identified in fecal pellets of black-tailed hares.

Common Name

Scientific Name

Shrubs

Big sagebrush

Rabbitbrush

Spiny hopsage

Artemisia tridentata

Chrysothamnus nauseosus

Grayia spinosa

Perennial Grasses

Squirreltail

Sandberg bluegrass

Needle-and-thread

Sitanion hystrix

Poa secunda

Stipa comata

Perennial forbs

Erigeron

Scurf pea

Balsamroot

Yarrow

Turpentine Cymopterus

Annual forbs

Tumble mustard

Spring draba

Blazing star

Matted cryptantha

Wing-nut cryptantha

Erigeron spp.

Psoralea lanceolata

Balsamorhiza careyana

Achillea lanuTosa

Cymopterus terebinthinus

Sisymbrium altissimum

Draba verna

Mentzalia sp.

Cryptantha circumscissa

Cryptantha pterocarya 
BNWL-1931

Special Distribution in

Category UC-70

\section{DISTRIBUTION LIST}

\section{OFFSITE}

1 ERDA Chicago Patent Group

A. A. Churm

4 ERDA Division of Biomedical and Environmental Research
J. L. Liverman
C. L. Osterberg
J. Swinebroad
R. L. Watters

ERDA Technical Information Center (Special)

1 Holifield National Laboratory

4 ERDA Division of Nuclear Fuel Cycle and Production

F. P. Baranowski

D. E. Saire

G. B. Pleat

W. R. Voight, Jr.

1 Argonne National Laboratory

9700 South Carr Avenue

Argonne, Illino is 60439

Librarian

1 Brookhaven National Laboratory

Research Library, Reference Section

Upton, L.I., New York 11973

1 E. I. duPont De Nemours and Co.

Aiken

Savannah River Laboratory

Technical Information Service

Room 773-A

Aiken, South Carolina 29801

1 Lawrence Radiation Laboratory

Lawrence Livermore Laboratory

Technical Information Dept., $L-3$

P. 0. Box 808

Livermore, California 94550 
2 Los Alamos Scientific Laboratory

P. 0. Box 1663

Los Alamos, New Mexico 87544

W. C. Hanson

T. Hakonson

1 Oak Ridge National Laboratory

Oak Ridge Operations Office

Oak Ridge, Tennessee 37830

S. I. Auerbach

1 ERDA Nevada Operations Office

P. 0. Box 14100

Las Vegas, Nevada 89114

P. Dunaway

Individuals in the United States

1

Dr. 0. Doyle Markham

Environmental Sciences Branch

Health Services Laboratory

USERDA

P. 0. Box 2108

Idaho Falls, Idaho 83401

1 Dr. Ward F. Whicker

Radiology and Radiation Biology Deoartment

Colorado State University

Fort Collins, Colorado 80521

1 Dr. David Willis, Chairman

Department of General Sciences

Oregon State University

Corvaliis, Oregon 97731

1 Dr. Mike Smith, Director

Savannah River Ecology Laboratory

Drawer $E$

Aiken, South Carolina 29801

ONSITE

1 . Patent Attorney 
ERDA Richland Operations Office
B. J. Melton/P. F. X. Dunigan
0. J. Elberg/D. M. Smith
F. R. Standerfer

J. D. Anderson

H. Babad

G. E. Backman

D. J. Brown

L. E. Bruns (3)

G. Burton, Jr.

F. R. Dornheim

D. Grudin

G. L. Hanson

M. K. Harmon

W. M. Harty

0. F. Hill

H. H. Hopkins, Jr.

R. E. Isaacson (5)

R. K. Lee

C. W. Malody

H. L. Maxfield

T. R. McKenzie

B. J. McMurray

D. C. Nelson

G. C. Oberg

K. R. Price

H. P. Shaw

R. M. Smithers

G. T. Stocking

V. A. Uresk (5)

W. J. Van Siyke

J. H. Warren

A. T. White

D. D. Wodrich

W. H. Zimmer

R. A. Zinsli
T. W. Ambrose
J. P. Corley
L. L. Eberhardt
W. H. Rickard (5)
W. L. Templeton (2)
C. M. Unruh 
Battelle-Northwest (Continued)

B. E. Vaughan (5)

E. L. Klepper (2)

J. F. Cline (2)

D. W. Uresk (5)

Biology Library (3)

Technical Information Files (3)

Technical Publications (1)

J. L. Helbling (1) 